\title{
Análise de técnicas de reconciliação de dados aplicadas a um sistema aquecedor de tanque agitado
}

\author{
Analysis of Data reconciliation techniques applied to a continuous \\ stirred tank heater
}

\author{
Felipe Santana Santos ${ }^{1}$; Leocarlos Bezerra da Silva Lima²
}

\begin{abstract}
Resumo
A medição das variáveis envolvidas em um processo industrial tem uma grande importância em sistemas em que decisões são tomadas baseadas nessas medições, como é o caso de sistemas de controle. Porém, essas medições sempre são acompanhadas de erros, sejam eles aleatórios ou grosseiros. No processo de aquecimento da água utilizando um sistema aquecedor de tanque agitado contínuo isso não é diferente. Uma alternativa para uma maior confiabilidade das variáveis é o uso da reconciliação de dados (RD). A RD trata de um problema advindo da evolução das técnicas de medição e armazenamento de dados. Esta tem o papel de garantir a consistência destes dados, utilizando a redundância das variáveis medidas e um modelo estatístico da medição para aumentar a precisão dos dados. Este trabalho investiga as técnicas de reconciliação de dados e seu emprego na redução dos erros observados nas medições num sistema aquecedor de tanque agitado contínuo.
\end{abstract}

Paravras chave: Erros aleatórios. Reconciliação de dados. Sistema aquecedor de tanque agitado contínuo.

\begin{abstract}
The measurement of variables from an industrial process is of great importance in systems in which decisions are made based on these measurements, as in control systems. However, these measurements are always accompanied by errors, random or gross. This is not different in the process of water heating. An alternative for a greater reliability of the variables is the use of data reconciliation (DR). DR arises from the development of techniques for measurement and data storage. Its role is to ensure the consistency of data using the redundancy of the measurements and a statistical model of the measurements to increase its accuracy. This work investigates techniques for data reconciliation and its use in reducing the errors observed in measurements on a continuous stirred tank heater.
\end{abstract}

Keywords: Random errors. Data reconciliation. Continuous stirred tank heater.

\footnotetext{
1 Mestrando em Engenharia Elétrica pela Universidade Federal de Sergipe; felipesanttana@hotmail.com

2 Docente do Departamento de Engenharia Elétrica da Universidade Federal de Sergipe; leocarlos@ufs.br
} 


\section{Introdução}

Para um bom funcionamento de qualquer sistema automatizado, seja estacionário ou dinâmico, na indústria química ou em sistemas robóticos, é imprescindível o emprego de um projeto de sistema de controle com auxílio de um modelo adequado do processo, e alimentado com medições confiáveis das variáveis de estado de interesse (FREIRE et al., 2008).

Os dados de um processo são obtidos através de instrumentos físicos e rotinas de medição de precisão finita. Em geral, erros de medição podem ser categorizados em duas classes (BENQLILOU, 2004):

1. Erros aleatórios: devidos a variações ou distúrbios de natureza não controlada. Ex.: ruído. São considerados independentes e com distribuição normal de média zero;

2. Erros não aleatórios ou grosseiros: são gerados por eventos não aleatórios, podendo estar relacionados com a medição (mau funcionamento dos instrumentos, por exemplo) ou com o processo (vazamentos, perdas, modelos inadequados ou imprecisos, etc.).

O emprego de dados corrompidos pode comprometer a atuação do controle do processo, podendo levar uma planta a operar num ponto subótimo ou inseguro, levar a perda de especificação de produtos, poluição ambiental, perdas financeiras ou altos custos de operação (MORAD; YOUNG; SVRCEK, 2005).

A chamada reconciliação de dados (RD) é uma técnica voltada à melhoria da confiabilidade das medições realizadas e constitui abordagem de filtragem baseada em modelos do sistema (TJOA; BIEGLER, 1991; DAROUCH; ZASADZINSKI, 1991; CROWE, 1996; ÖZYURT, 2004; BAI; THIBAULT; MCLEAN, 2006). As técnicas de RD baseiam-se na redundância das medidas e em restrições impostas por modelos do sistema.
Dependendo das restrições do modelo do processo, a RD pode ser estacionária ou dinâmica, como também linear ou não linear (BENQLILOU, 2004). Esta pode ainda possibilitar a redução da frequência de calibração de sensores e a diminuição de erros nas medições, tornando o monitoramento de plantas mais preciso para tomada de decisão (LAWRANCE, 1989).

Objetiva-se aqui analisar as técnicas de reconciliação de dados e seu emprego na redução dos erros observados nas medições num sistema aquecedor de tanque agitado contínuo (THORNHILL; PATWARDHAN; SHAH, 2008).

A próxima seção descreve algumas técnicas de RD. A seção 3 descreve o sistema aquecedor de tanque agitado contínuo. Na seção 4 apresenta os resultados obtidos em simulação para as técnicas abordadas. Na quinta seção, são descritas as conclusões do trabalho.

\section{Reconciliação de Dados}

Erros aleatórios são causados por ruídos e não podem ser totalmente eliminados. Considerando que estes erros são provenientes de um número suficientemente grande de diferentes fontes, o teorema central do limite aplica-se (BAGAJEWICZ; JIANG, 1997) e a soma destes distúrbios tende à distribuição gaussiana. Assim, erros aleatórios são modelados como ruído branco gaussiano $\mathrm{N}\left(0, \sigma^{2}\right)$.

Erros grosseiros geralmente são decorrentes de possíveis falhas num determinado equipamento de medição. Tais erros também podem ser considerados com função densidade de probabilidade gaussiana, porém com média diferente de zero $\mathrm{N}\left(\beta, \sigma^{2}\right)$ (TJOA; BIEGLER, 1991). Por este motivo, o efeito prejudicial deste tipo de erro é muito maior do que o do aleatório.

O problema de $\mathrm{RD}$ pode ser visto como um problema de minimização do erro médio quadrático entre as variáveis medidas e as 
descritas por um modelo (FARIAS, 2009). Este problema tem como restrições modelos do processo que podem ser: lineares, bilineares ou não-lineares, assim como estáticos ou dinâmicos. As técnicas de RD foram criadas baseadas nestas restrições e podem ser divididas em dois grandes grupos: reconciliação de dados estacionária (RDE) e reconciliação de dados dinâmica (RDD).

Sistemas dinâmicos são representados por equações diferenciais que descrevema dependência das variáveis do processo no tempo. De modo geral, sistemas dinâmicos são representados por equações algébrico-diferenciais, com restrições não lineares e muitas vezes com parâmetros e condições iniciais desconhecidas (PRATA, 2009). Os problemas de RDD são mais complexos que os de RDE, porém a RDD apresenta um número maior de aplicações na indústria, em que os processos estão frequentemente variando suas características.

O presente trabalho emprega três técnicas de RDD a um sistema aquecedor de tanque agitado contínuo: filtro de Kalman (FK), filtro de Kalman estendido (FKE) e filtro de Kalman Unscented (FKU).

\section{Filtro de Kalman}

Stanley e Mah (1977) propuseram o uso de filtro de Kalman (FK) como solução do problema de RDD. Essa técnica iterativa é útil em modelos dinâmicos lineares para reconciliar e/ou estimar dados. Ela exige que o erro tenha média zero, ou seja, que não seja grosseiro.

O FK possui duas fases: predição e atualização. A fase de predição usa a estimativa do estado da iteração anterior para produzir uma estimativa do estado na iteração atual. Na fase de atualização, a medição da iteração atual e a covariância do erro são usadas para melhorar a medição e chegar a uma estimativa mais precisa do estado. Seguem as equações que o descrevem.

$$
\begin{aligned}
& \hat{x}_{k}^{-}=A \hat{x}_{k-1}^{-}+B u_{k}, \\
& \underbrace{P_{k}^{-}=A P_{k-1} A^{T}+Q}_{\text {Predição }},
\end{aligned}
$$

$K_{k}=P_{k}^{-} H^{T}\left(H P_{k}^{-} H^{T}+R\right)^{-1}$,

$\hat{x}_{K}=\hat{x}_{k}^{-}+K_{k}\left(z_{k}-H \hat{x}_{k}^{-}\right)$,

$$
\underbrace{P_{K}=\left(I-K_{k} H\right) P_{k}^{-},}_{\text {Atualização }}
$$

em que $\mathrm{A}, \mathrm{B}$ e $\mathrm{H}$ são matrizes determinísticas com dimensões apropriadas, o índice $\mathrm{k}$ representa os estados, $K_{k}$ o ganho de Kalman, $z_{k}$ o valor medido, $\hat{x}_{k}^{-}$a estimação do estado a priori, $u_{k}$ é o vetor com as variáveis de entrada, $\hat{x}_{k}$ a estimação do estado a posteriori, I a matriz identidade, $P_{k}$ a matriz de covariância do erro, Q é a covariância do ruído do estado e $\mathrm{R}$ é a covariância do ruído da medição.

\section{Filtro de Kalman Estendido}

O FK na forma que foi proposto inicialmente não apresenta bons resultados em sistemas não lineares. Para emprego em tais sistemas, Jang, Joseph e Mukai (1986) propuseram o chamado filtro de Kalman estendido (FKE). Este usa uma formulação iterativa e sequencial do problema de estimação baseada numa descrição linearizada do modelo dinâmico. Tal linearização é feita analiticamente ou numericamente com o auxílio de matrizes jacobianas. As equações do FKE são descritas por:

$$
\begin{aligned}
& \hat{x}_{k}^{-}=f\left(\hat{x}_{k-1}, u_{k}\right), \\
& \underbrace{P_{k}^{-}=F_{k} P_{k-1} F_{k}^{T}+Q,}_{\text {Predição }} \\
& K_{k}=P_{k}^{-} H_{k}^{T}\left(H_{k} P_{k}^{-} H_{k}^{T}+R\right)^{-1}, \\
& \hat{x}_{K}=\hat{x}_{k}^{-}+K_{k}\left(z_{k}-h\left(\hat{x}_{k}^{-}\right)\right), \\
& \underbrace{P_{K}=\left(I-K_{k} H_{k}\right) P_{k}^{-},}
\end{aligned}
$$


sendo:

$$
\begin{gathered}
\left.\frac{\partial}{\partial}\right|_{\hat{x}_{k}} u_{k} \\
H_{k}=\left.\frac{\partial h}{\partial x}\right|_{\hat{x}_{k}^{-}},
\end{gathered}
$$

em que $\mathrm{F}_{\mathrm{K}}$ e $H_{\mathrm{K}}$ são as matrizes jacobianas, o índice $\mathrm{k}$ representa os estados, $K_{k}$ o ganho de Kalman, $z_{k}$ o valor medido, $\hat{x}_{k}^{-}$a estimação do estado a priori, é o vetor com as variáveis de entrada, $\hat{x}_{k}$ a estimação do estado a posteriori, I a matriz identidade, $P_{k}$ a matriz de covariância do erro, $\mathrm{Q}$ é a covariância do ruído do estado e $\mathrm{R}$ é a covariância do ruído da medição.

\section{Filtro de Kalman Unscented}

Os erros observados no FKE são devidos às linearizações realizadas para o cálculo da média e da covariância de uma variável aleatória. O filtro de Kalman unscented (FKU) evita estes erros utilizando a transformação unscented (UT). Basicamente, $2 \mathrm{~L}+1$ sigma-pontos (L é a dimensão do estado) são escolhidos baseadosnadecomposiçãoda raizquadrada da covariância anterior (JULIER; UHLMANN, 2004). Estes sigma-pontos são propagados através da verdadeira função não-linear $f$, sem aproximação, e em seguida uma média ponderada e uma covariância são calculadas. As equações do FKU são, então, calculadas pelo procedimento a seguir.

Inicialização:

$$
\begin{aligned}
\hat{x}_{k-1}^{a} & =\left[\begin{array}{lll}
x_{k} & v_{k} & n_{k}
\end{array}\right]^{T}, \\
P_{k-1}^{a} & =\left[\begin{array}{ccc}
P_{k-1 \mid k-1} & 0 & 0 \\
0 & Q & 0 \\
0 & 0 & R
\end{array}\right] .
\end{aligned}
$$

$$
\text { Cálculo dos sigma-pontos } \chi_{k-1}^{a} \text { : }
$$

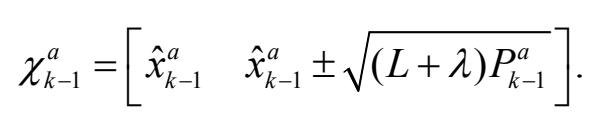

Atualização (os sigma-pontos se propagam através da função $f$ ).

$$
\chi_{k \mid k-1}^{a}=f\left(\chi_{k-1}^{a}, u(k)\right) .
$$

Utilizando somas ponderadas o cálculo de $x_{k}^{-} \mathrm{e}$ $P_{k}^{-}$é feito por:

$\hat{x}_{k}^{-}=\sum_{i=0}^{2 L} W_{i}^{m} \chi_{i, k \mid k-1}$,

$$
P_{k}^{-}=\sum_{i=0}^{2 L} W_{i}^{c}\left[\chi_{i, k \mid k-1}-\hat{x}_{k}^{-}\right]\left[\chi_{i, k \mid k-1}-x_{k}^{-}\right]^{T} .
$$

Do mesmo modo, define-se o vetor de inovações do processo $\hat{y}_{k}^{-}$:

$$
\begin{aligned}
& I_{k \mid k-1}=h\left(\chi_{k \mid k-1}, u(k)\right), \\
& \hat{y}_{k}^{-}=\sum_{i=0}^{2 L} W_{i}^{m} I_{i, k \mid k-1} .
\end{aligned}
$$

A covariância das medições $P_{y_{k}, y_{k}}$ é calculada da seguinte forma:

$$
P_{y_{k}, y_{k}}=\sum_{i=0}^{2 L} W_{i}^{c}\left[I_{i, k \mid k-1}-\hat{y}_{k}^{-}\right]\left[I_{i, k \mid k-1}-\hat{y}_{k}^{-}\right]^{T} .
$$

A correlação cruzada $P_{x_{k}, y_{k}}$ entre os estados estimados e a sequência de medições é: 


$$
P_{x_{k}, y_{k}}=\sum_{i=0}^{2 L} W_{i}^{c}\left[\chi_{i, k \mid k-1}-\hat{x}_{k}^{-}\right]\left[I_{i, k \mid k-1}-\hat{y}_{k}^{-}\right]^{T} .
$$
forma:

O ganho $K$, então, é calculado da seguinte

$$
K=P_{x_{k}, y_{k}}\left(P_{y_{k}, y_{k}}\right)^{-1} \text {. }
$$

Os valores de $\hat{x}_{K}$ e $P_{K}$, então, são calculados:

$$
\begin{aligned}
& \hat{x}_{K}=\hat{x}_{k}+K\left(y_{k}-\hat{y}_{k}^{-}\right), \\
& P_{K}=P_{k}^{-}-K P_{y_{k}, y_{k}} K^{T},
\end{aligned}
$$

sendo $x_{k}, u_{k}, v_{k}$ e $n_{k}$ os vetores de estado, das entradas, do ruído do processo e do ruído das medições, respectivamente, no instante $k . P_{k-1 \mid k-1}$ é a covariância do erro, Q a covariância do ruído do processo, R é a covariância do ruído da medição e I é a matriz identidade. $W^{m}$ é um peso para as medidas e $W^{c}$ um peso para a covariância, ambos calculados na transformação unscented, $\lambda$ é um valor escalar também calculado na UT.

\section{Sistema Aquecedor de Tanque agitado contínuo}

O modelo utilizado nesse trabalho foi proposto por Thornhill, Patwardhan e Shah (2008). Este consiste num sistema aquecedor de tanque agitado contínuo, do inglês continuous stirred tank heater (CSTH).

No sistema CSTH, água quente e fria são misturadas, então aquecidas por uma bobina de aquecimento e drenadas do tanque através de um duto. Na figura 1, pode-se observar a configuração deste processo.
Figura 1. Sistema Aquecedor de Tanque Agitado contínuo

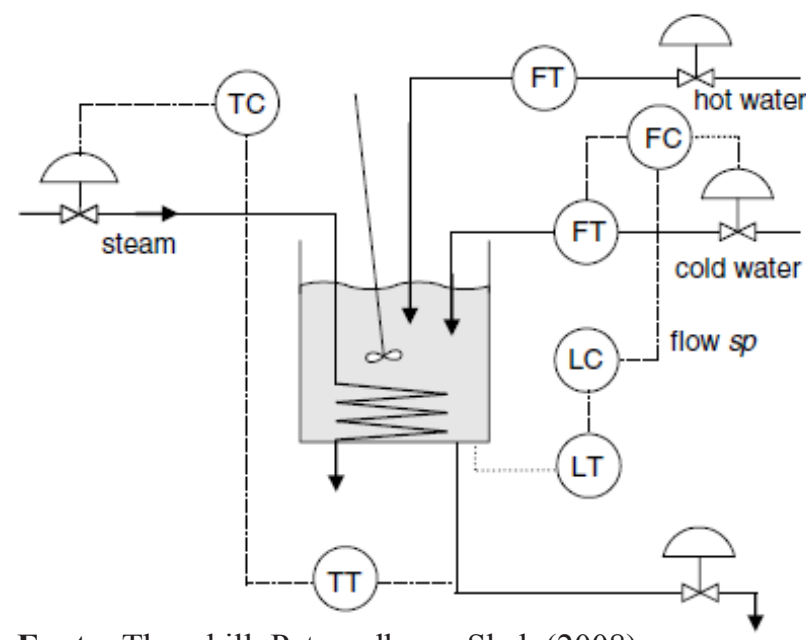

Fonte: Thornhill, Patwardhan e Shah (2008).

Uma característica valiosa deste modelo é que utiliza ruído e perturbações medidas (não simuladas) e, por conseguinte, fornece uma plataforma realista para utilização da RD.

A variável monitorada neste trabalho foi a temperatura da água do tanque. O ruído da temperatura foi monitorado com a metade do tanque cheio e em circuito fechado com a temperatura controlada. O ruído tem a maioria dos componentes de alta frequência. Algumas componentes de média e baixa frequência também podem ser vistas. A figura 2 apresenta o modelo implementado no Simulink.

\section{Resultados}

As técnicas de reconciliação de dados apresentadas foram implementadas e simuladas usando Matlab/Simulink. Um modelo preciso do sistema aquecedor de tanque agitado contínuo implementado por Thornhill, Patwardhan e Shah (2008) em Matlab/Simulink foi utilizado. As variáveis controladas neste aquecedor são o fluxo de água fria, o nível de água misturada e a temperatura da água do tanque. Dentre elas, a RD será aplicada à temperatura (variável temp). 
Figura 2. Sistema aquecedor implementado em Simulink.

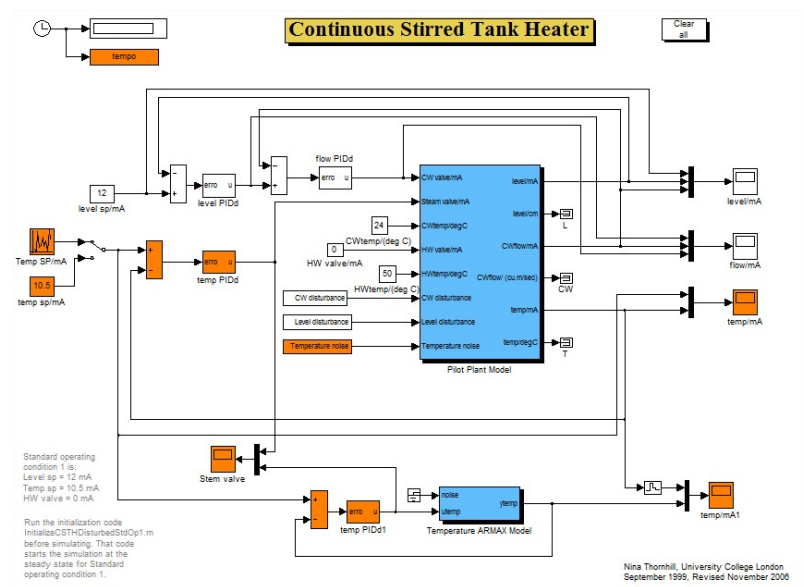

Fonte: Adaptado de Thornhill, Patwardhan e Shah (2008).

A comparação entre as técnicas de $\mathrm{RD}$ foi feita com base no erro médio quadrático entre as variáveis reconciliadas e as variáveis originais (não corrompidas pelo ruído), para um mesmo nível de degradação das medições pelo ruído. Através da figura 3, verifica-se que o comportamento da variância do erro médio quadrático (EMQ) é linear em relação ao aumento da variância do ruído presente nas variáveis. Isso implica que, independentemente da magnitude do ruído, a técnica de RD irá atenuar o mesmo de modo proporcional. Conclui-se que o EMQ constitui uma figura de mérito adequada para uma comparação da eficácia entre diferentes técnicas de RD na presença de ruído gaussiano.

Figura 3. Variância do ruído $x$ variância do EMQ para o FK.

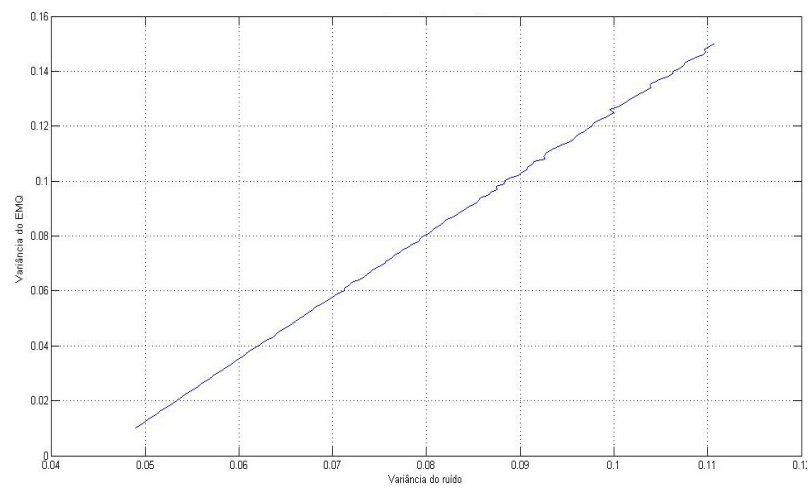

Fonte: Os autores.

\section{Filtro de Kalman}

Aplicou-se o FK à variável temp. Observando a figura 4, pode-se notar que o FK apresentou um bom resultado durante os períodos de estabilidade, porém nos momentos de mudança brusca da temperatura (mudança de set point) o resultado não foi tão satisfatório. Este resultado já era esperado, visto que o FK não apresenta bons resultados sob condições de não linearidade.

Figura 4. Variável temp reconciliada empregandose o filtro de Kalman.

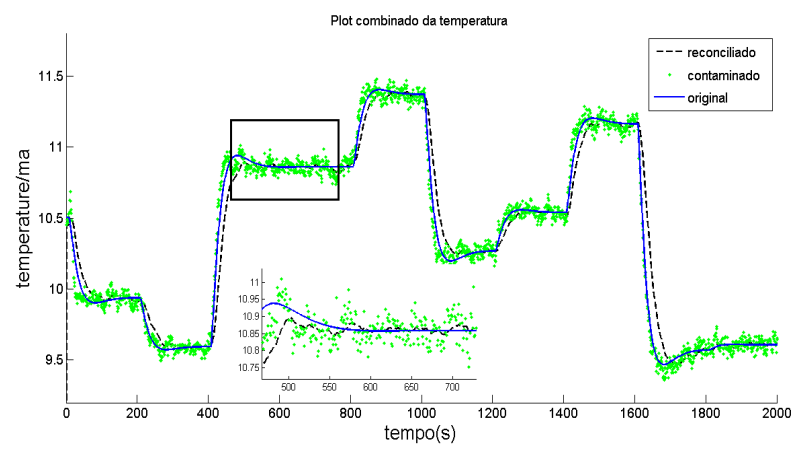

Fonte: Os autores.

$\mathrm{Na}$ figura 5, observa-se o EMQ da variável reconciliada ilustrada na figura 4. Como pode-se ver, há momentos em que o EMQ apresenta piora significativa. Isto deve-se a variações abruptas da temperatura provocadas por mudanças no set point do controlador. A variância do erro médio quadrático associado à temperatura foi de .

Figura 5. Erro médio quadrático associado à variável temp utilizando-se o FK.

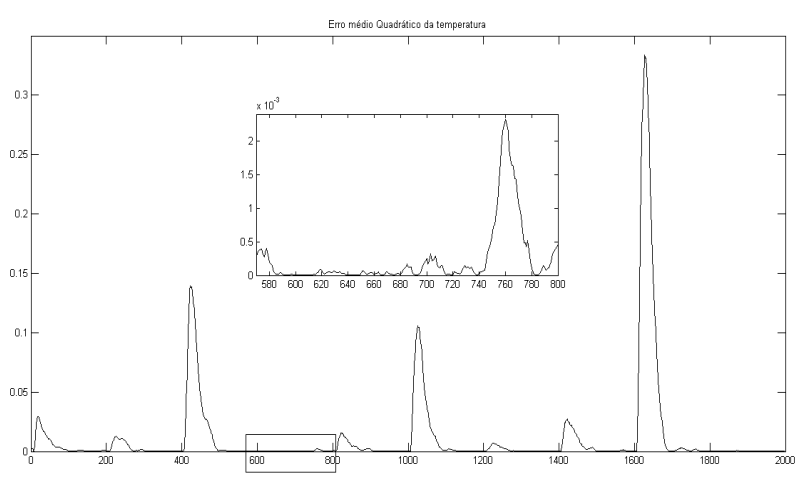

Fonte: Os autores. 


\section{Filtro de Kalman Estendido}

Aplicou-se o filtro de Kalman estendido à variável temp. Na figura 6 , observa-se que o filtro apresentou um ótimo resultado, inclusive nos momentos de variação brusca. $\mathrm{O}$ resultado condiz com os trabalhos presentes na literatura, pois o FKE foi criado justamente para suprir a deficiência do FK sob condições de não linearidade.

Figura 6. Variável temp reconciliada empregandose o filtro de Kalman estendido.

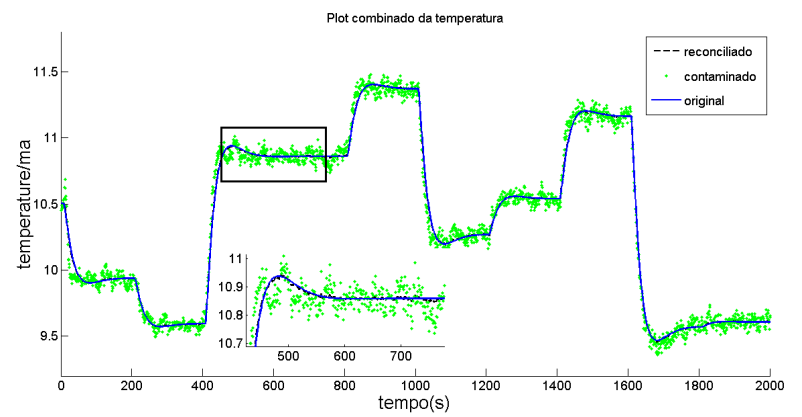

Fonte: Os autores.

A figura 7 ilustra o EMQ associado à temperatura. A variância do EMQ obtido foi de $6,1612 \cdot 10^{-9}$.

Figura 7. Erro médio quadrático associado à variável temp utilizando-se o FKE.

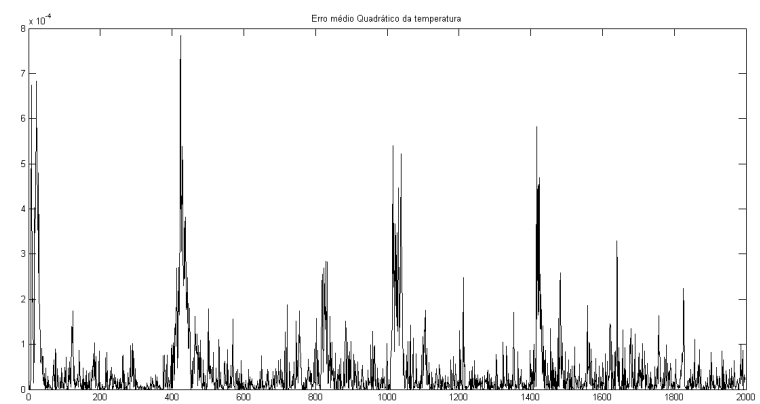

Fonte: Os autores.

\section{Filtro de Kalman Unscented}

Aplicou-se o filtro de Kalman unscented à variável temp. Na figura 8 , observa-se que o filtro apresentou um excelente resultado, inclusive nos momentos de variação brusca. $\mathrm{O}$ resultado foi tão satisfatório que fica difícil distinguir a variável sem ruído da variável reconciliada. Este excelente resultado foi devido à robustez do FKU a não linearidades.

Na figura 9, observa-se o EMQ da variável da temperatura ilustrada na figura 8 . A variância do EMQ obtido foi de $2,1724.10^{14}$.

\section{Comparação entre os métodos escolhidos}

Na tabela 1, observa-se que o uso do filtro de Kalman unscented fez com que o resultado da reconciliação fosse otimizado, quando comparado aos outros dois métodos. Esse resultado mostra que o uso de tal técnica tem um desempenho melhor, mesmo na presença de variações abruptas. Também pode-se observar que o resultado do filtro de Kalman estendido foi melhor do que o FK.

Figura 8. Variável temp reconciliada empregandose o filtro de Kalman unscented.

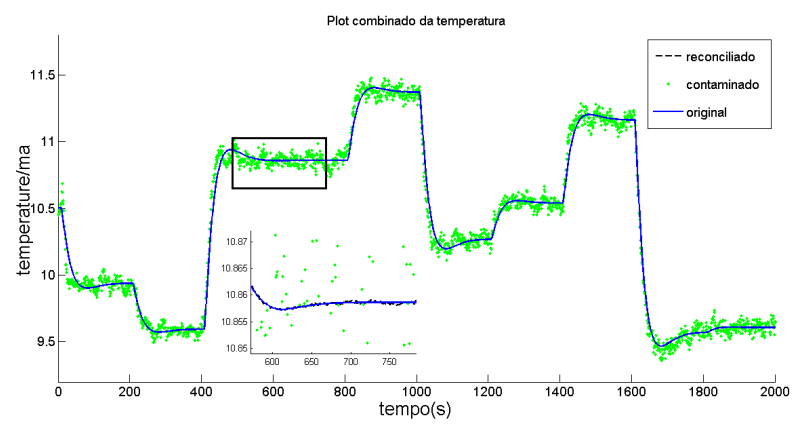

Fonte: Os autores.

Figura 9. Erro médio quadrático associado à variável temp utilizando-se o FKU.

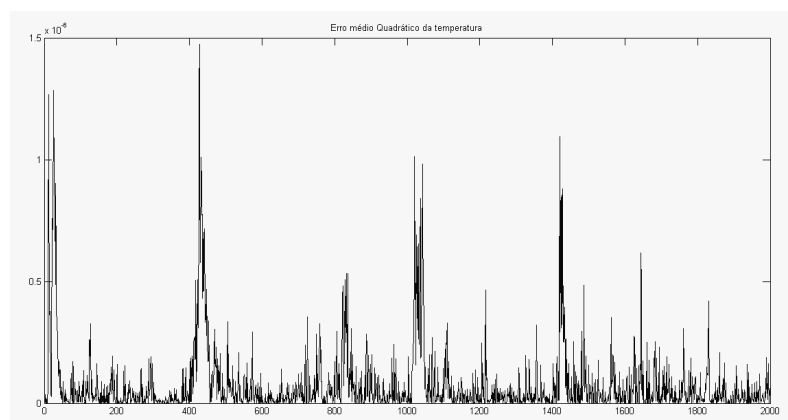

Fonte: Os autores. 
Tabela 1 - Variâncias dos erros médios quadráticos da temperatura empregando filtro de Kalman (FK), filtro de Kalman estendido (FKE) e filtro de Kalman unscented (FKU).

\begin{tabular}{|c|c|}
\hline & Var (EMQ p/ temp) \\
\hline FK & 0,0016 \\
\hline FKE & $6,1612.10^{-9}$ \\
\hline FKU & $2,1724.10^{-14}$ \\
\hline
\end{tabular}

Fonte: Cálculos realizados pelos autores no Matlab.

O melhor desempenho do FKU comparado ao FKE e ao FK deu-se devido a dois fatores: o aumento da precisão do tempo de atualização e a melhoria da precisão da covariância (KANDEPU; FOSS; IMSLAND, 2008).

\section{Conclusões}

Nesse trabalho foram avaliadas três técnicas para a reconciliação de dados: o filtro de Kalman, o filtro de Kalman estendido e o filtro de Kalman unscented. Estas técnicas foram aplicadas a uma variável do aquecedor de tanque agitado contínuo, a temperatura da água do tanque. Com base nos resultados obtidos, verificou-se que as três técnicas apresentaram bom resultado na reconciliação dos dados analisados do aquecedor. O método utilizado para comparação de algoritmos foi o do erro médio quadrático entre as variáveis reconciliadas e as variáveis originais, para um dado nível de degradação das variáveis pelo ruído. Através do mesmo, verificou-se que o uso do FKU apresentou significante melhor resultado dentre as três técnicas utilizadas.

O presente trabalho pode ser complementado pela utilização de técnicas de tratamento de erros grosseiros visto que, na ocorrência destes, a reconciliação de dados torna-se ineficaz. Essas técnicas podem ser implementadas antes da reconciliação de dados, como prevenção da aplicação desta na presença de erros grosseiros, ou utilizando estatística robusta, em que a reconciliação de dados e o tratamento de erros grosseiros são realizados simultaneamente.

\section{Agradecimentos}

À Coordenação de Pesquisa -- COPES da Universidade Federal de Sergipe -- UFS pelo financiamento de bolsa de pesquisa e ao prof. Oscar Alberto Zanabria Sotomayor, do Departamento de Engenharia Elétrica -- DEL da UFS, pelas importantes contribuições ao trabalho.

\section{Referências}

BAGAJEWICZ, M. J.; JIANG, Q. Integral approach to plant linear dynamic reconciliation. AIChE Journal, v. 43, n. 10, p. 2546-2558, Oct 1997.

BAI, S.; THIBAULT, J.; MCLEAN, D. D. Dynamic data reconciliation: Alternative to kalman filter. Journal of Process Control, v. 16, n. 5, p. 485-498, Jun 2006.

BENQLILOU, C. Data reconciliation as a framework for chemical processes optimization and control. 2004. Tese (Doutorado em Engenharia Química) - Universitat Politécnica de Catalunya.

CROWE, C. M. Data reconciliation - progress and challenges. Journal of Process Control, v. 6, n. 2, p. 89-98, 1996.

DAROUCH, M.; ZASADZINSKI, M. Data reconciliation in generalized linear dynamic systems. AIChE Journal, v. 2, n. 37, p. 193-201, 1991.

FARIAS, A. C. Avaliação de estratégias para reconciliação de dados e detecção de erros grosseiros. 2009. Dissertação (Mestrado emEngenharia Química) - Universidade Federal do Rio Grande Do Sul. 
FREIRE, E. O.; CARVALHO, E.A. N.; CARDOSO, C. A. V.; LUCIANO, B. A. Execution monitoring applied to data estimation processes. In: LATIN AMERICAN ROBOTICS SYMPOSIUM (JRI 2008 - EnRILARS2008). Salvador, BA, Brasil: [s.n.], 2008.p. 97-102.

JANG, S.; JOSEPH, B.; MUKAI, H. Comparison of two approaches to on-line parameter and state estimation of nonlinear systems. Industrial Enginnering and Chemical Process Design Development, v. 25, p. 809-814, July 1986.

JULIER, S. J.; UHLMANN, J. K. Unscented filtering and nonlinear estimation. Proceedings of the IEEE, v. 92, n. 3, p. 401-422, 2004.

KANDEPU, R.; FOSS, B.; IMSLAND, L. Applying the unscented kalman filter for nonlinear state estimation. Journal of Process Control, v. 18, p. 753-768, 2008.

LAWRENCE, P. J. Data reconciliation: Getting better information. Hydrocarbon Processing, v. 68, p. 55-60, 1989.

MORAD, K.; YOUNG, B. R.; SVRCEK, W. Y. Rectification ofplant measuremrnts using a statical framework. Computers \& Chemical Engineering, v. 29, n. 5, p. 919-940, April 2005.

ÖZYURT, R. W. P. D. B. Theory and practice of simultaneous data reconciliation and gross error detection for chemical processes. Computers \& Chemical Engineering, v. 28, n. 3, p. 381-402, 2004.

PRATA, D. M. Reconciliação robusta de dados para monitoramento em tempo real. 2009. Tese (Doutorado em Engenharia Química) -UFRJ/ COPPE, Rio de Janeiro.

STANLEY, G.; MAH, R. Estimation of flows and temperatures in process networks. AIChE Journal, v. 23, p. 642-650, Sep 1977.

THORNHILL, N. F.; PATWARDHAN, S. C.; SHAH, S. L. A continuous stirred tank heater simulation model with applications. Journal of Process Control, v. 18, p. 347-360, 2008.
TJOA, I. B.; BIEGLER, L. T. Simultaneous strategies for data reconciliation and gross error detection of nonlinear systems. Computers \& Chemical Engineering, v. 15, n. 10, p. 679-690, 1991. 
